\title{
Reviews in Medicine
}

\section{Infectious diseases}

\author{
P.D. Welsby \\ Department of Infectious Diseases, City Hospital, 51 Greenbank Drive, Edinbrugh EH10 5SB, UK
}

\section{Introduction}

Infection is relevant to so many specialties that it is unrealistic to provide a comprehensive review: I have therefore focused on the more important or interesting aspects.

Although human immunodeficiency virus (HIV) and antibiotics have been covered in other reviews I have mentioned various aspects that seem to have general relevance to infectious diseases.

\section{Antibiotics and gastroenteritis}

Norfloxacin, a new quinolone, has activity against most common gastrointestinal pathogens including Salmonella, Shigella and Campylobacter. Norfloxacin or placebo was given to patients over the age of 12 who had acute diarrhoea of presumed infective aetiology for 5 or fewer days. ${ }^{1}$

Overall norfloxacin had a favourable effect when compared with placebo as it reduced the intensity and to some extent the duration of symptoms, but the effect was attributable to the subgroup of patients from whom enteropathogenic bacteria were isolated or who were severely ill. Norfloxacin seemed to delay the elimination of Salmonella and to induce resistance in Campylobacter. Much the same comments can be made about most antibiotics - there is little indication for routine antibiotics in the routine case of suspected infection with enteropathogenic bacteria.

Four reasons have been given for not using antibiotics in bacterial diarrhoea: they are ineffective, they prolong excretion of the bacteria concerned, they may encourage emergence of bacterial resistance and there will be side effects.

In patients with positive bacteriology, ciprofloxacin is no more effective than placebo in reducing the duration of diarrhoea, although interestingly ciprofloxacin reduces the duration of diarrhoea slightly in all patients with 'gastroenteritis'. The benefit of quinolones seems modest at best. If fluoroquinolones were used to treat each case of gastroenteritis in the UK then about $13,000 \mathrm{~kg}$

Correspondence: P.D. Welsby, F.R.C.P. (Ed.) would be needed each year (no wonder that drug companies are interested in this particular market!). Antibiotics can be used to prevent travellers' diarrhoea but quinolones are no more effective than other classes of antibiotics.

The topic of quinolones for salmonella gastroenteritis is taken up in depth in a paper headed 'for debate' by Wilcox and Spencer. ${ }^{2}$ In 1990 in the United Kingdom there were about 26,000 human cases of salmonella infection, double the number reported 5 years previously.

If patients have longstanding excretion of salmonellae which is unacceptable (mostly on occupational grounds) the quinolones are a useful treatment option. Because excretion of salmonellae after gastroenteritis may be intermittent, fairly intensive stool culture follow-up is necessary before the true effect of treatment can be assessed.

Development of antibiotic resistance of faecal flora has not to date been observed with quinolones and the side effects of quinolone treatment appear to be few.

Wilcox and Spencer do not reach a conclusion but hope that the points they raise will stimulate discussion. In the meantime I feel that routine use of antibiotics to prevent gastroenteritis cannot be recommended. An exception would be when important people briefly visit at-risk areas of the world to fulfil important functions (the author of this review would of course include himself in this category!).

\section{Bovine spongiform encephalopathy}

New cases of this encephalitis are being reported in cattle at the rate of 500 weekly, and between 1986 and 1989 the parts of such cattle would have been entering the food chain as offal. So far no association has been found between disease in cattle and the disease in humans. A similar disease in sheep, scrapie, has not spread to humans (yet, or as far as we know?) and thus the provisional omens for humans are good, but spongiform encephalopathies have appeared in species previously unaffected. 
The spongiform encephalopathies are initiated by deposition of an abnormal form of protein in the brain: the protein is genetically determined but not all cases could arise by spontaneous mutation. An infective particle of some sort may be responsible and, if it is, how species restricted will it (or similar mutations) be? A useful editorial ${ }^{3}$ concludes that no evidence exists to deny that the chances of spongiform encephalopathy causing human disease are extremely small - but neither can the possibility be dismissed. Because of the long incubation period we shall have to wait and see.

\section{Chlamydia: an extended role?}

Chlamydia pneumoniae is a well-recognized respiratory pathogen causing epidemics every 4-5 years or so and antibodies are found in about $50 \%$ of middle-aged adults, ${ }^{4}$ but it may also have a role to play in atherosclerotic blood vessel disease. A paper suggested that chronic chlamydia infection, as evidenced by elevated $\operatorname{IgA}$ titres and immune complexes (which were found before myocardial infarction) was an independent risk factor for coronary heart disease. ${ }^{5}$

Chlamydia pneumoniae may have a role in ear, nose and throat infections. Antibody studies suggested recent infection in $2 / 19$ patients with sinusitis, 10/52 with tonsillitis, $8 / 34$ with otitis media and 6/33 with laryngitis ${ }^{6}$ suggesting that Chlamydia pneumoniae is an important and common upper respiratory pathogen.

\section{Curiosities}

Curiosities are always memorable no matter how dubious their relevance may seem. The most famous curiosity was the irritating inhibition of bacterial growth on fungally contaminated culture plates which resulted in the identification of penicillin. Apparently there are dangers from the humble guppy that graces every tropical aquarium! A paper from Trinidad ${ }^{7}$ tells us that guppies have been used as a biological means to control Aedes aegypti in water tanks used for storing drinking water. These insects may transmit yellow fever and dengue, and guppies have an appetite for these mosquitos. But guppies may harbour pathogens: studies indicate that they carry and can excrete potential pathogens including Pseudomonas aeruginosa and Escherichia coli. Obviously this has relevance for those who store water in insectexposed areas but, for those of us who keep tropical fish, this is (another) reason why we should wash our hands after dealing with fish tanks.

\section{The role of endotoxins in septic shock}

Endotoxin, a lipopolysaccharide part of the outer membrane of Gram-negative bacteria, is often blamed for causing, or at least triggering, most of the manifestations of septicaemic shock. It is known that this occurs in animals but does it also apply in humans? The answer is yes. A laboratory worker gave himself $1 \mathrm{mg}$ of Salmonella minnesota endotoxin (3,750 times the dose used in volunteer studies) as a misguided treatment for a recently diagnosed tumour. ${ }^{8} \mathrm{He}$ developed high cardiac output hypotension with lowered systemic vascular resistance and had symptoms consistent with generalized capillary-leak syndrome with noncardiogenic pulmonary oedema, abnormal renal and hepatic function, and disseminated intravascular coagulation. It thus seems certain that monoclonal antibodies (vide infra), if they can neutralize endotoxin effectively, will have a role in the management of endotoxic shock.

\section{Health of health care workers}

The potential infectivity of HIV, especially to health care workers, is always a worry. A useful editorial outlines the risks for surgeons and starts off, like all good stories, with an arresting first sentence which compels a casual browser to read on: 'Sex has seriously complicated surgery during recent years'.?

Two-tier precaution systems (differentiation between what the clinician perceives are 'low' and 'high' risk patients) are reasonable in low prevalence areas but in high prevalence areas adoption of universal precautions (that is, in all patients) is a safer option. The risk to surgeons is small: ${ }^{10}$ most health care workers who have seroconverted have sustained accidents using hollow needlestick exposures to HIV-positive blood and even then, the seroconversion rate is about one in 300 of such exposures ${ }^{11}$ (surgeons frequently have non-hollow needlesticks but there seems to be little evidence of surgically acquired HIV infection).

Apart from one 'highly curious' incident in which an HIV-positive dentist infected several patients there is little evidence that a health care worker has transmitted infection to a patient. Nevertheless, HIV is almost always going to be a life-shortening infection and, predictably, there will be repeated attempts to make sure, by mandatory testing, that there is no possibility that a surgeon is HIV positive.

Like all good stories the reader is left with a question to worry about - 'Would surgeons accept a situation where they are expected to have mandatory routine (HIV) tests, which might result in 
the loss of their surgical work, while they are not allowed to carry out routine screening HIV antibody tests on their patients?' A Joint Working Party has issued comprehensive guidelines. ${ }^{12}$

\section{Hepatitis A}

Hepatitis A virus was propagated in cell culture in 1978, and formalin-inactivated and attenuated vaccines have been developed. An inactivated virus vaccination against hepatitis $A$ is now available (HAVRIX).

There are numerous questions that have to be asked of any new vaccine. The answers, as far as is known concerning this particular vaccine, are given in parenthesis.

Is the disease severe or common enough to attempt vaccination? (Certainly, but only in certain groups including travellers from the developed world who visit developing countries and in whom vaccination is an alternative to human pooled immunoglobulin. Vaccination can be used, in association with pooled human immunoglobulin, if there is an ongoing outbreak of hepatitis A because there is only a slight decrease in mean anti-hepatitis A virus titres following immunization in those also given immunoglobulin. The incidence of travelrelated hepatitis $A$ is uncertain but in $198814 \%$ of reported cases (566) of hepatitis A were travel related and no fewer than 5.04 million UK residents travelled to areas where vaccination is recommended. In 1990 passive prophylaxis with immunoglobulin was given to an estimated 600,000 persons. Thus, the incidence of clinical hepatitis $A$ in returning UK travellers seems to be moderately low. $)^{13}$

Approximately 30-35 million travellers from industrialized nations visit developing countries where their risk of developing symptomatic hepatitis A is 3-6 per thousand per month of stay if they remain unprotected. ${ }^{14}$

Does the vaccine store well? (Yes). And does it require a 'cold chain' of transportation? No. What are the side effects? (Very few, all trivial). ${ }^{15}$

What is the ideal age for vaccination and can the vaccine be given effectively and safely in other age groups if need be? (Research is proceeding and the vaccine almost certainly will be safe in all age groups but the dose in those less than 16 years is not yet established.)

Does the vaccine protect against different serotypes of heptatitis A virus? (Yes).

What dose is required? (The dosage is 720 ELISA units given intramuscularly spaced 2 weeks to 1 month apart. If persistent immunity for up to 10 years is desired then a further booster dose would be required to maintain anti-hepatitis $\mathbf{A}$ virus antibody titres within protective levels). ${ }^{16}$
What proportion of those vaccinated respond? (Detectable antibody develops in $97 \%$ after the initial vaccination and $100 \%$ after the second dose $^{17}$ and if antibody equates with protection then $97 \%$ should be protected 1 month after the first injection. Coincidental administration of human pooled immunoglobulin should be given to those going to be exposed to high-risk situations within a month), although coincidental administration results in a two-fold lower antibody titre than administration of hepatitis $A$ vaccine alone, and thus a booster dose may be required sooner. ${ }^{18}$

By what route should it be given? (Intramuscular).

Can the vaccine be usefully used post-exposure? (Probably, if given early enough, but simultaneous immunoglobulin would also be indicated).

How long does protection last? (Unknown probably a long time).

Do antibody levels reflect protection and do these need to be monitored and if so how often? (Almost certainly antibody levels do reflect protection but the other answers will be known in the future).

Is it effective to screen for pre-existent immunity and only vaccinate the non-immunes? (This depends on the prevalence of natural immunity in a defined population and the future risk of infection).

Can the vaccine be given along with other established vaccines and is there any decline in efficacy of any of the constituent vaccines? (Seemingly yes and no, respectively. Certainly hepatitis B and hepatitis $A$ vaccine can be given together with no decline in efficacy of either). ${ }^{19}$

Who could be vaccinated, and who should be vaccinated? (The answer will depend on variables including hepatitis A epidemiology in various countries and their financial resources: selective vaccination of high-risk adults would not be expected to lower the overall incidence of infection in most countries). Travellers to underdeveloped countries should be vaccinated - those staying in 'renowned' hotels have a hepatitis A attack rate of about 3-6/1,000 per month of stay and if they stay in situations of poor hygiene the rate is about $20 / 1,000$ per month. (Workers in childcare centres and sanitation workers could also be considered for vaccination, as could male homosexuals ${ }^{20}$ ).

Can high prevalence countries afford routine vaccination? (Probably not. Additionally in most developing countries hepatitis $\mathbf{A}$ is a relatively minor childhood disease, vaccination of children may raise the mean age of infection and hepatitis $A$ is more severe in post-childhood sufferers).

Can vaccination be used to cut short epidemics? (In 'single source group infections' once clinical cases are apparent the answer is no, but if continued case-to-case spread is occurring then the answer is probably yes.)

What proportion of the population need to be 
vaccinated in order to eliminate the infection? (Unknown. The proportion required need not be $100 \%$ as any vaccine that caused an average case to transmit infection on average to less than one other person would cause the disease to die out).

What will be the cost? (Relatively expensive. At the time of writing in Britain the cost is $£ 13.60$ per dose).

Is manufacture of the vaccine going to be commercially viable? (Yes).

What is the cost per case prevented and is this cost effective - including direct and indirect costs of illness? (The cost will differ in different countries, for example, in some Northern European countries up to $20 \%$ of fulminant viral hepatitis is caused by hepatitis $A^{21}$ but in Britain the percentage is generally much lower, although occasional reports have suggested a higher incidence in certain areas. A London-based study in 1983 revealed that, of 73 cases of fulminant hepatitis, $31.5 \%$ were caused by hepatitis A.) $)^{22}$

\section{Hepatitis B}

Should surgeons who test positive for the e antigen of hepatitis B be routinely transferred to low-risk duties? This thorny question is usefully addressed in a 'for-and-against' written debate..$^{23}$

'For' is an occupational health doctor and 'against' is a gynaecological surgeon. Predictably surgeons, including cardiovascular surgeons and gynaecological surgeons, are much more likely to spread infection than other health care professionals.

Several preliminary facts. The risk of a single percutaneous exposure to ' $e$ ' hepatitis B-positive blood transmitting infection is up to $30 \%$. A little recognized problem for surgeons is that properly conducted vaccination programmes aimed at protecting surgeons will indirectly (but inevitably) also screen for those who are already hepatitis B positive because those surgeons who fail to respond with production of hepatitis B antibody after vaccination will either be non-responders to vaccination or will be those who have no antibody because their (unsuspected) hepatitis B positivity means that any antibody will be 'mopped-up'. Because proper vaccination programmes should include post-vaccination assessment of antibody positivity the non-responders will either have to be investigated further or presumed (rightly or wrongly) to be immune despite their failure to respond to vaccination.

Against the proposal, or rather expressing reservations, was the gynaecological surgeon who was understandably much concerned with compensation and retraining for those found to be positive.

\section{Historical lessons}

Humanity appears to need plagues to form a focus for group concern and to provide a role for central government, but the memory of groups and governments means that each generation has to relearn from its own mistakes. Syphilis was rampant in the Belle Glade area of Florida (near the euphonious Lake Okkechobee) in the 1940s. Other diseases of poverty and poor hygiene, including tuberculosis and typhoid, were also common. A paper $^{24}$ details the factors that led to an unusually high prevalence of syphilis and the social interventions that were attempted to bring it under control led to the recognition that socioeconomic conditions are major factors in the epidemiology (and therefore prevention) of most infectious diseases. Similar factors are now causing a very high prevalence of HIV and acquired immunodeficiency syndrome (AIDS) in Belle Glade. Doctors, if they are to care for the individual patient and also care for the good of populations will also have to give advice regarding the introduction of socioeconomic improvements to improve the general health of certain sections of society. But will governments listen?

\section{Human herpesvirus type 6}

Human herpesvirus 6 (HHV-6) was initially isolated from mononuclear cells from six adults who had lymphoproliferative disorders ${ }^{25}$ and various studies have shown that most children have been infected by the age of three, ${ }^{26}$ but until the association of this infection with exanthem subitum (roseola) had been made ${ }^{27}$ it was unclear whether any illness resulted. A further investigation has revealed that this virus is a major cause of acute febrile illnesses in young children ${ }^{28}$ Fourteen per cent of children in the first two years of life presenting to an Accident and Emergency Department during a 6 month period had acute viraemic HHV-6 infection. Only a few had illnesses resembling exanthem subitum: most had several days of fever, often strikingly high when compared with children of the same age with other febrile illnesses.

\section{Legionnaires' disease}

More evidence is accumulating that identification of a single patient with Legionnaires' disease should initiate specialist epidemiological seeking of other cases because there may well be a continuing source of infection. Investigations covering 15 Health Boards in Scotland ${ }^{29}$ revealed evidence of 
numerous clusters of infection, all but two previously unrecognized.

\section{Malaria}

A total of 2,332 cases of malaria were imported into the United Kingdom in 1991 and there were 12 deaths, 11 of which were caused by Plasmodium falciparum infection. ${ }^{30}$

The conventional wisdom is that uncomplicated non-falciparum malaria is unlikely to be fatal. The one death in a non-falciparum infection occurred in a patient with ovale malaria who had a ruptured spleen. The author reviewer now begs leave to pronounce one of his firmly held beliefs - no one should ever feel for a spleen! The relaxed palpating hand should be progressively moved from the right lower quadrant towards the left upper quadrant whilst the patient is taking moderate breaths and the spleen allowed to come and palpate the waiting fingers (how many spleens are ruptured by overvigorous palpation is of course unknown, but all will be labelled as 'spontaneous rupture').

Over $90 \%$ of cases of falciparum malaria were contracted in Africa and over $80 \%$ of vivax malaria cases were contracted in South Asia. The failure of many patients to take prophylaxis was again apparent. The incubation periods of malaria usually apply to those who are not immune and who have taken no prophylaxis. Eighty-nine per cent of falciparum patients presented within the first month of arrival in the UK $(99 \%$ within 6 months) but only a quarter of vivax malaria presented within the first month of arrival. One encouraging factor is that the increase in cases of falciparum malaria from Kenya (a popular tourist destination) was not as steep as the rise in the number of UK travellers visiting that country. The malarial prophylaxis that should be given in areas where chloroquine resistance is common is a matter of importance.

Does a rising parasite count provide a prediction of failure in the treatment of falciparum malaria? A small study ${ }^{31}$ revealed that parasite counts rise in the first 12 hours in both successful and unsuccessful treatment. Rising counts thereafter were rarer in treatment successes. The relative risks of treatment failure was 13.7 if the parasite count was higher at 48 hours than on admission and 19.4 if higher at 60 hours. The relative risk of failure was 3.8 if the count was higher at 24 hours than 12 hours. These are useful figures if one is treating with drugs in the (perhaps erroneous) assumption that the malaria is sensitive.

It is always pleasant to have one's beliefs confirmed. I always tell patients who have developed malaria despite claiming to have taken their prophylaxis appropriately that the pro- phylaxis had caused their malaria to be less severe than it might have been. A study based on imported malaria in the Hospital for Tropical Diseases in London concluded that prior chemoprophylaxis reduced the severity of malaria. ${ }^{32}$

In the Gambia it appears that malaria chemoprophylaxis given during pregnancy would reduce infant mortality by about one fifth in primigavidae but by less than $5 \%$ in multigravidae. Thus it seems that, in malaria endemic areas, primagravidae should be given malaria prophylaxis for the sake of their children as well as for their own sake. ${ }^{33}$

A total of 145,003 travellers returning from East Africa completed an in-flight questionnaire and a subsequent questionnaire 3 months later. Of the 139,164 who were staying in East Africa for less than one year, 296 developed malaria (275 caused by $P$. falciparum). In those who took no prophylaxis the incidence of falciparum malaria was $1.2 \%$ per month of stay. Prophylactic effectiveness was $91 \%$ for those who took mefloquine, $82 \%$ for pyrimethamine and sulphadoxine, $72 \%$ for chloroquine plus proguanil and $10-42 \%$ for chloroquine at various doses. Mefloquine was thus the most effective prophylaxis for short-term visitors. ${ }^{34}$ The current recommendations for malarial prophylaxis have been well reviewed. ${ }^{35}$

\section{Meningitis}

Serogroup B Neisseria meningitidis is the most common cause of epidemic meningococcal diseases in developed countries. Unfortunately the usual vaccines only protect against group $A$ and $C$ infections. A case control study of a Group B vaccine revealed efficacy of between $74 \%$ in those aged 48 months or older, $47 \%$ in those aged $24-47$ months but less than $37 \%$ in those less than 24 months old. ${ }^{36}$ The vaccine under test was thus effective only in older children and adults. Other trials of other vaccines are in progress and no doubt vaccines including Group B will become available soon.

Bacterial meningitis is a disease, like infective endocarditis, that has changed its nature over the last few decades consequent to changes in invasive medical technology, antibiotic usage and the population. A review of 493 episodes of meningitis in adults, albeit from a tertiary referral centre, revealed much interesting information. ${ }^{37}$ Fortythree per cent of episodes were nosocomial, mostly related to neurosurgical interventions. In the community-acquired infections the most common pathogen was Streptococcus pneumoniae (37\%), followed by Neisseria meningitidis (13\%) and Listeria monocytogenes $(10 \%)$. Nine per cent of all patients had recurrent meningitis, often associated with cerebrospinal fluid leakage. The overall 
fatality rate was $25 \%$ and did not vary over the 27 year period of study.

Only two-thirds of patients had fever, neck stiffness and altered mental state, but all had at least one of these $(88 \%$ had neck stiffness and, contrary to previous reports, the elderly were no less likely to have neck stiffness). A rash was present on admission in $11 \%$ and, interestingly, a petechial rash also was apparently present in some patients with pneumococcal meningitis. A petechial rash was also seen in staphylococcal meningitis, but this is not surprising - petechial rashes are not uncommon in staphylococcal septicaemia, particularly if associated with underlying endocarditis.

The incidence of nosocomial and recurrent meningitis was high, but this was probably related to the tertiary nature of some of the admissions. The static mortality rate over 27 years was interesting. The reviewer suspects that the benefit in diagnosing and treating meningitis was matched by increases in patient vulnerability (including age and neurosurgical interventions).

\section{Monoclonal antibodies}

Controversies still rage concerning the usefulness of currently available Gram-negative endotoxin antibody. One product (HA-1A) was withdrawn at the end of 1992 because of the increased mortality of those who received it but did not have Gramnegative sepsis.

One review article ${ }^{38}$ provided information that there were about 500,000 cases of sepsis each year in the USA with an estimated mortality of $35 \%$, half being caused by Gram-negative bacilli with blood cultures being positive in half of these. Of the blood culture-positive patients $25 \%$ die of complications directly attributable to the bacteraemia and $10 \%$ from the underlying disease. Steroids or naloxone have shown no consistent efficacy but what is the situation with monoclonal antibody, specifically HA-1A? The author's estimate was that, at best, $10 \%$ of patients with sepsis would have improved survival if either of two currently available products were used wisely, and with HA-1A the cost per year of life saved would be $\$ 24,100$. But if everyone with sepsis were to receive monoclonal antibody then the extra health bill would be 2,000 million dollars. The author then outlines the various options that could be adopted towards use of monoclonal antibodies. His conclusion was that they help some patients but they are of marginal benefit in practice.

A following 'Sounding Board' paper ${ }^{39}$ suggested that a second placebo-controlled trial was warranted (but this does not help us now). There were problems with the studies performed on HA-1A. A significant benefit of HA-1A was found in only one of many overlapping subgroups. The benefit was marginal. The beneficial effect on mortality was only seen at Centres with high mortality rates. The APACHE II system used to stratify patients may have been inappropriately applied. Patients who received inadequate or unknown antibiotic regimes were included. Finally the results were not stratified according to the time elapsed before the antibody or placebo was given. The Federal Drug Administration analysts had also suggested that, because multiple comparisons were made in the analysis, the value at which statistical significance should have been accepted should have been reduced to between 0.01 and 0.03 . Accordingly, HA-1A was only associated with beneficial outcomes (in terms of mortality from all causes) within a 28 day period in patients who did have Gram-negative bacteraemia (overall HA-1A was not effective in those clinically diagnosed as having Gram-negative sepsis). The authors concluded that the currently available evidence that HA-1A reduced mortality among patients with Gram-negative bacteraemia was suggestive but not conclusive.

I nearly always read medical journals backwards: the correspondence columns almost invariably provide much of interest and occasional amazement. Five weeks later I was not disappointed. ' $\$ 24,100$ per year of life saved compares very favourably with many other accepted current practices $^{\text {'40 }}$ and 'I want it for my patients' ${ }^{41}$ followed by an editor's note that the correspondent concerned held stock in the company that makes HA-1A.

The correspondence was shown to the original authors of the second paper who additionally commented that there had been a revised analytical plan in the original study which could have had potential for introduction of bias. Finally the author of the first paper entered the moral minefield by asking at what level of cost does a treatment become too high. ${ }^{42}$

This is all good controversial material which does not help us now. The British government have managed this problem by giving clinicians budgets and letting them make decisions. ${ }^{43}$

Can the current author help physicians now with their expensive dilemma? The answer is no. However, I am awaiting the results of HA-1A in meningococcal infection as this is the only Gramnegative bacteraemia that can be reliably diagnosed clinically at an early stage when treatment with HA-1A might be expected to be highly effective.

\section{New technology investigations}

For simple physicians there is a helpful review article $^{44}$ which explains the theory behind modern 
technology investigations. Use of polymerase chain reaction amplification is proving a particularly useful tool in identifying the nature of organisms that can be seen but not cultured. The organisms associated with bacillary angiomatosis, peliosis hepatitis and certain bacteraemias have yielded to such approaches.

\section{Notification of infectious diseases}

Notification of infectious diseases is important so that early knowledge of outbreaks can be obtained and appropriate actions taken at an early stage if necessary, and so that official records are available so that success of various interventions, including vaccination campaigns, can be assessed. Some infectious diseases are remarkable by the fact that they are not statutorily notifiable: syphilis and Legionnaires' disease are curiously notifiable but (for reasons that will forever remain essentially unreasonable to this writer) AIDS, or rather HIV, is not. A fascinating short paper ${ }^{45}$ asks how much do doctors know about the statutory requirement to notify infectious diseases. The answer is not enough, or more scientifically, there is a range between $14 \%$ (hospital doctors' awareness that mumps should be notified) and $96 \%$ (general practitioner's awareness that bacterial meningitis should be notified). Only $57 \%$ of general practitioners and $65 \%$ of hospital doctors thought that Lassa fever was notifiable. Forty-one per cent of general practitioners and $45 \%$ of hospital doctors (wrongly) thought that AIDS was statutorily notifiable. Plainly statutory notifications only provide a guide to the incidence of infectious diseases. The process of infectious disease notification is under review. ${ }^{46}$

This reviewer would like to see suspicion of certain illnesses (including bacterial meningitis, meningococcal septicaemia, and Lassa fever) statutorily notified by telephone as urgent authoritative action should be taken without any delay.

\section{Occupational diseases}

Infectious diseases have many fascinations, but one of the major ones is exactly how people manage to acquire their infections: often it seems that organisms are innocent parties which become pathogenic because humans seek them out. Wooley was a male white rhinoceros who had the misfortune to develop, amongst other symptoms, rhinorrhoea. Seven out of 24 zookeepers were subsequently shown to have been infected with Mycobacterium bovis from their charge $!^{47}$

\section{Opportunistic infections in AIDS}

Many opportunistic infections in AIDS are reactivations of pathogens acquired previously. This statement begs the question 'Which organisms can persist for many years and be reactivated when immunodeficiency occurs?' Leishmaniasis may be one such infection which may persist despite conventionally adequate treatment. Mice were treated with either thermotherapy or antimony to achieve clinical cure of Leishmania mexicana infection ${ }^{48}$ but lesions recurred after subsequent immunosuppression with cyclophosphamide or hydrocortisone. There are obvious implications for AIDS patients who have had leishmaniasis in the past or who have been in endemic areas.

Because the major defect in HIV infections is of cell-mediated immunity, infections with intracellular pathogens (mostly bacteria and viruses) predominate. However, the same principles may apply to other parasites. Hospital patients with AIDS had fewer Entamoeba coli and E. histolytica infections than would have been expected. ${ }^{49}$ The authors concluded that, whilst AIDS predisposes to isosporiasis and cryptosporidiosis, it did not predispose to infections with extracellular intestinal parasites such as Entamoeba species, Ascaris and Necator.

Hospitals are strange places to send patients. In particular patients with infections are admitted and so are patients who are particularly vulnerable to infections. AIDS units of course provide both types of patients; indeed individual patients often are both donors and recipients of infections. The quesiton arises 'can immunosuppressed patients transmit Pneumocystis carinii infection to each other?' The authors of a paper ${ }^{50}$ detail five (HIV negative but immunosuppressed) renal transplant patients who attended the same outpatient facility as AIDS patients and developed Pneumocystis carinii pneumonia (PCP). Studies on three patients with matched controls revealed that these three patients had more outpatient clinic encounters with AIDS patients who had presented, or subsequently developed, PCP. This suggests that AIDS patients developing PCP may transmit the infection to other immunosuppressed patients. The potential implications - increased need for separation of immunosuppressed patients - are worrying.

\section{Perils of research}

The lives of unusual people always make interesting reading and doctors are no exception. A brief account of the life of Howard Taylor Rickets $(1871-1910)^{51}$ concludes with the interesting information that he died of typhus whilst investigating an outbreak of typhus in Mexico. Stanislav 
von Prowazek, another typhus investigator, also died of typhus. Their immortality is perpetuated because, quite literally, when their disease was found to be caused by a distinct organism it was then called Rickettsia prowazekii.

\section{Polio}

Paralysis is known to be worse if people receive injections during the period of polio infection before the paralytic phase. A study in India ${ }^{52}$ demonstrated the effect of prior injections on the pattern and severity of paralytic polio. After injection there was a greater likelihood of death or lack of muscle strength recovery. Three-quarters of children studied had received unnecessary injections and of these $60 \%$ had more severe paralysis, and a non-paralytic attack became paralytic in $40 \%$. The authors rightly conclude that oral medicines rather than injections would reduce the incidence and severity of paralytic polio.

\section{Prevention of infection}

The risks of transfusion-acquired infection are declining progressively. The risk of transfusionacquired hepatitis $C$ in the USA has decreased markedly since the implementation of routine donor screening for antibodies and surrogate markers to hepatitis $\mathrm{C}$ virus. ${ }^{53}$ An accompanying leading article $\mathrm{e}^{54}$ broadens the discussion away from hepatitis $C$. The incidence of transfusion-related infections has declined because of three factors. Physicians have restricted the use of transfusion to where necessary and have increased the use of autologous transfusions and intra-operative blood salvage. Selection of donors is utilized. Every blood donation in the USA is screened for no less than seven infections (syphilis, hepatitis B, hepatitis C, HIV types 1 and 2, and human T-cell lymphotropic viruses type 1 and 2). Selected specimens are also tested for cytomegalovirus and pooled-plasma products undergo viral inactivation procedures. But rest assured the microbes will come up with something new!

With increasing public awareness of certain disease there will be demands for post-exposure treatments from those with possible, probable, and highly unlikely exposures to the relevant pathogen. Should all those with needlestick exposure to HIV-positive material receive zidovudine prophylaxis? The answer is not known: a double-blind placebo-controlled study was terminated when it was realized that data showing efficacy could not result, and because of the low rate of seroconversion and reluctance of those involved to receive placebo.
Lyme disease has a high media profile especially in areas of countries where ticks are known to be infected with Borellia burgdorferi. Should those with tick bites in such areas be given antibiotic prophylaxis? A controlled trial in an area of America where Lyme disease is endemic ${ }^{55}$ concluded that, even in such areas, the risk of infection after a recognized deer-tick bite is so low that prophylactic antimicrobial treatment is not routinely indicated.

\section{Prions}

Prions (proteinaceous infectious particles) are controversial, undoubtedly pathogenic, protein structures which are devoid of nucleic acids but which, nevertheless, function like micro-organisms to cause infectious diseases. They display a high level of resistance to standard sterilizing procedures. But are prions organisms or no more than transmissable toxic proteins that accumulate and thereby damage host cell tissue? ${ }^{56}$

Eight prion diseases are currently recognizable. ${ }^{57}$ In animals prions are found in scrapie, transmissable mink encephalopathy, bovine spongiform encephalopathy, and the chronic wasting disease of mule deer and elk. In humans, prions are found in kuru (transmitted by cannibalism), CreutzfeldJacob disease (usually sporadic), GerstmannStraussler-Scheinker syndrome and fatal familial insomnia. Some of these illnesses are transmitted naturally, some are inherited and some are both. All are long incubation period diseases which cause degeneration of the central nervous system without inflammatory reaction, or obvious humoral or cellular reactions in the blood.

Although the human diseases are uncommon, there are similarities between prion disease and common human diseases such as Alzheimer's disease.

\section{Resistant Staphylococcus aureus}

Although the problems in the UK have been relatively minor, hospitals in certain parts of the world have been plagued with methicillin-resistant Staphylococcus aureus (MRSA) infections. In Japan this organism is epidemic and attributed to overprescribing of third-generation cephalosporins (which selected out MRSA against which they have no activity). The real reason seems to be that Japanese hospitals are paid a fixed official price for each antibiotic they prescribe, but the drug companies often offer the hospitals discounts which provides hospitals with extra income, rumoured to be a total of $£ 7$ billion each year. 


\section{Rubella vaccination}

In the United Kingdom immunization rates are high and the uptake of measles, mumps and rubella vaccine, which was introduced in October 1988, has been dramatic. The number of (recognized) rubella infections in pregnancy was 12 in 1991 and two in 1992. There have only been 94 births of congenitally infected babies since 1987, of which only 19 occurred in 1990-1992. Twenty-two mothers giving birth to congenitally infected babies were either Asian or Oriental, and three at least acquired their infections abroad. ${ }^{58}$ Perhaps immunization should be offered to immigrants upon arrival?

\section{Sarcoidosis}

Sarcoidosis is one of several diseases about which everything is known apart from the cause and the cure. Sarcoidosis has many of the characteristics of an immune reaction to an infection and the noncaseating granulomas found in sarcoidosis may perhaps be a reaction to mycobacterial infections. Sixteen patients with sarcoidosis had biopsies and Mycobacterium tuberculosis DNA was detected in seven, but in only one of the matched controls. ${ }^{59}$

Not surprisingly, two of four specimens came from patients known to be infected with Mycobacterium tuberculosis were positive. The conclusion is that Mycobacterium tuberculosis DNA is detected as readily in sarcoidosis as in patients with tuberculosis and that infection with $\mathrm{Myco}$ bacterium tuberculosis may be linked to the cause of sarcoidosis. There are problems because certain non-pathogenic mycobacteria contain similar DNA sequences.

However, the advocacy of a large controlled trial of anti-tuberculous therapy in sarcoidosis is premature ${ }^{60}$ because a large number of patients would have to be recruited, there would have to be much more substantial evidence of a pathogenic role for mycobacteria.

\section{Sexual behaviour}

The evidence is that unsafe sexual behaviour is increasing after a period of decline and recent HIV transmissions may disproportionately affect younger men. ${ }^{61}$

The possible decline in safe sex, associated with oversaturation of warning messages, or almost psychopathic disregard of such messages, has profound significance for the epidemiology of many sexually transmitted diseases. Surprisingly, health education messages which elicit high anxiety do not work in the long term for most people in the population. This disappointing fact partially explains the increase in other sexually transmitted diseases.

In Britain the number of new patients seen at sexually transmitted disease clinics has risen from 1,670 to 1,950 per 100,000 between 1981 and 1990 . Such statistics are plainly worrying especially now that HIV is a 'routine sexually transmitted disease'. There is an increasing accumulation of circumstantial evidence that Kaposi's sarcoma has a sexually transmitted cofactor in those who develop AIDS. The major suspicion arises because Kaposi's sarcoma is rare in HIV acquired by intravenous drug abuse, whereas it is relatively common in those who acquire HIV homosexually (presumably because of the greater number of partners on average that gay men have compared to intravenous drug abusers and/or heterosexuals). A paper from Canada identified two risk factors (both more common in homosexual male groups) for Kaposi's sarcoma high numbers of sexual partners and use of nitrite inhalants. ${ }^{62}$ The former suggest a sexually related factor, presumably an infectious agent, that might possibly be treatable or preventable.

\section{Tuberculosis}

The Americans are having problems with multidrug-resistant tuberculosis, with several large outbreaks. ${ }^{63-65}$

Such infections are not only a problem for the infected patient: infection is transmissable to others including healthcare workers (eight, of whom four were HIV positive, in the hospitals reporting) and about 50 healthcare workers at another hospital had tuberculin skin test conversions following exposure to hospitalized patients with multidrugresistant tuberculosis. Nearly all patients had organisms resistant to rifampicin and isoniazid.

Multidrug-resistant tuberculosis requires prolonged treatment and even so the 'cure' rate is poor. The mortality rates of multidrug-resistant tuberculosis of the infected patients was extraordinarily high and their progression to death may be rapid. In one study ${ }^{66}$ only about half of patients with tuberculosis resistant to rifampicin and isoniazid eventually had negative cultures despite carefully selected regimens administered for extended periods.

HIV-positive patients who are infected with Mycobacterium tuberculosis develop clinical illness more rapidly than HIV-negative patients. Treatment of multidrug-resistant tuberculosis is difficult in patients with HIV infection because of the underlying immunosuppression and the fact that a proportion of those with HIV belong to groups in which compliance is traditionally poor.

The measures required to contain multidrugresistant patients include measures to improve 
compliance (notably observed ingestion of therapy) and negative air pressure isolation for those hospitalized. The authors of an accompanying editorial $^{67}$ conclude that successful control of multidrug-resistant tuberculosis will require rapid, concerted action and close collaboration among a variety of governmental and private agencies. The author of this review wonders which of these admirable modes of action will turn out to be a euphemism for 'legally enforcable'.

In the UK we have a policy of using BCG immunization to prevent natural primary complex tuberculosis: therefore we are nearly all Mantoux positive and need to be screened with chest $\mathrm{X}$-rays. The Americans in contrast do not use BCG and rely upon skin test conversions to identify those who have been infected.

Most, but not all, of those who have primary complex infection with Mycobacterium tuberculosis will recover spontaneously and thereafter permanently suppress their infection unless they become immunosuppressed by diseases including HIV. It will be interesting to see if AIDS-related tuberculosis in the UK will be less of a problem than in the USA and, if it is, whether BCG will deserve credit. One also wonders if the Americans will change their policy towards BCG.

The diagnosis of tuberculosis is easy if the organism is plentiful and it can be easily seen and grown. However, if the organisms are scanty and grow slowly then other techniques such as the polymerase chain reaction (PCR) may be useful. But this may be too expensive and technically difficult for use in developing countries (where tuberculosis is also staging a resurgence). A short paper $^{68}$ gives an example of how sophisticated techniques like PCR can be modifed and simplified for use in the tropics.

\section{Vaccination}

Most vaccinations are, and should be termed, immunizations! ${ }^{69}$

Vaccination means stimulation of immunity to smallpox by inoculation of live vaccinia virus whereas immunization refers to the stimulation of immunity by deliberate intervention without causing the disease in question. The British Health Education Authority avoids the terms vaccination and vaccine. The author of the letter requests everyone else to do the same. However, whilst agreeing with the historical justification, I am not optimistic!
Although typhoid is a well-publicized risk for travellers, the number of infections is low, partially because of 'pure water paranoia' and partially because of vaccination (immunization!). The old vaccine was a heat-killed whole organism vaccine (effectiveness about $50-90 \%$ but there were discouraging side effects) but recently a live attenuated orally administered vaccine (protection about $90 \%$ ) and a parenterally administered Vi-antigen vaccine (protection about $60-70 \%$ ) have become available.

Most vaccines have been evaluated in endemic areas and little or no information is available for visitors from non-endemic areas (it would need huge trials because of the very low proportion of those who would be expected to develop typhoid fever). A useful editorial ${ }^{70}$ concluded that the only differences between the vaccines are the routes of administration, dose schedules and adverse effects.

\section{Vitamin A}

A meta-analysis of 20 controlled trials ${ }^{71}$ has shown a reduction in overall mortality, and a reduction in deaths from diarrhoeal diseases and respiratory diseases in those given vitamin A. The authors concluded that 'an adequate supply of vitamin A has a major role in preventing mortality and morbidity in developing countries. In developed countries vitamin A may also have a role in those with life-threatening infections such as measles'. Unfortunately the mechanism for these reductions in morbidity and mortality is incompletely resolved'. Such mechanisms, once discovered, may have wider significance in the development of anti-infective agents.

\section{Whooping cough}

It is often assumed that named organisms are homogeneous. It is important to realize that the individual members of populations of organisms, even of named organisms, may differ in important characteristics. A paper ${ }^{72}$ reports that multiple strains of Bordetella pertussis circulate in a given human population at one time.There is also considerable variation in the population of strains between countries. These findings plainly have implications for vaccine development - all, or at least most, Bordetella organisms should be discouraged by a new vaccine. 


\section{References}

\section{Antibiotics and gastroenteritis}

1. Winstrom, J., Jertborn, M. \& Ekwall, E. Empiric treatment of acute diarrhoeal disease with Norfloxacin. Ann Intern Med 1992, 117: 202-208.

2. Wilcox, M.H. \& Spencer, R.C. Quinlones and salmonella gastroenteritis. J Antimicrob Chemother 1992, 30: 221-228.

Bovine spongiform encephalopathy

3. Harrison, P.J. \& Roberts, G.W. How now mad cow? Br Med $J$ 1992, 304: 929-930.

Chlamydia: an extended role?

4. Grayston, J.T., Campbell, L.A., Kuo, C.C. et al. A new respiratory tract pathogen: Chlamydia pneumoniae strain TWAR. J Infect Dis 1990, 161: 618-625.

5. Saikku, P., Leinonen, M., Tenkanen, L. et al. Chronic Chlamydia pneumoniae infection as a risk factor for coronary heart disease in the Helsinki Heart Study. Ann Intern Med 1992, 116: 273-278.

6. Hashiguchi, K., Ogawa, H. \& Kazuyama, Y. Seroprevalence of Chalmydia pneumoniae infections in otolaryngeal diseases. J Laryng Otol 1992, 106: 208-210.

\section{Curiosities}

7. Chadee, D.D. Bacterial pathogens isolated from guppies (Poecilia reticulata used to control Aedes aegypt in Trinidad. Trans R Soc Trop Med Hyg 1992, 86: 693.

\section{The role of endotoxins in septic shock}

8. Da Silva, A.M.T., Kaulbach, H.C., Chuidian, F.S., Lambert, D.R., Suffredini, A.F. \& Danner, R.L. Brief report: shock and multiple organ dysfunction after self-administration of Salmonella endotoxin. $N$ Engl J Med 1993, 328: 1457-1460.

\section{Health of health care workers}

9. Shanson, D.C. Current surgical controversies over HIV infection. J Hosp Infect 1991, 17: 77-81.

10. Gazzard, B.G. \& Wastell, C. Editorial. HIV and Surgeons. $\mathrm{Br}$ Med J 1990, 310: 1003-1004.

11. Marcus, R. and the CDC Cooperative Needlestick Surveillance Group. Surveillance of health care workers exposed to blood from patients infected with human immunodeficiency virus. $N$ Engl J Med 1988, 319: 1118-1123.

12. Joint Working Party of the Hospital Infection Society and the Surgical Infection Study Group. Risks to surgeons and patients from HIV and hepatitis; guidelines on precautions and management of exposure to blood or body fluid. $\mathrm{Br} \mathrm{Med}$ $J$ 1992, 305: 1337-1343.

\section{Hepatitis A}

13. Black, M.E., Begg, N. \& Behrens, R.H. Hepatitis A vaccination. Lancet 1992, 340: 244

14. Steffen, R. Risk of hepatitis A in travellers. Vaccine 1992, 10 (Suppl 1): S69-S72.

15. Andre, F.E., D'Hondt, E., Delem, A. \& Safary, A. Clinical assessment of the safety and efficacy of an inactivated hepatitis A vaccine: rationale and summary of findings. Vaccine 1992, 10 (Suppl 1): S160-S168.

16. Wiedermann, G., Ambrosch, F., Andre, F.E., D'Hondt, E., Delem, A. \& Safary, A. Persistence of vaccine-induced antibody to hepatitis A virus. Vaccine 1992, 10 (Suppl 1): S129-S131.

17. Tilzey, A.J., Palmer, S.J., Barrow, S. et al. Clinical trial with inactivated hepatitis $\mathbf{A}$ vaccine and recommendations for use. Br Med J 1992, 304: 1272-1276.

18. Leentvaar-Kuijpers, A., Coutinho, R.A., Brulein, V. \& Safary, A. Simultaneous passive and active immunisation against hepatitis A. Vaccine 1992, 10 (Suppl 1): S138-S141.
19. Ambrosch, F., Andre, F.E., Delem, A. et al. Simultaneous vaccination against hepatitis $A$ and $B$ : results of a controlled study. Vaccine 1992, 10 (Suppl 1): S142-S145.

20. Atkins, M., Zambon, M. \& Watkins, P. Should susceptible homosexual men be offered immunisation? Br Med J 1993, 307: 562 .

21. O'Grady, J. Management of acute and fulminant hepatitis A Vaccine 1992, 10 (Suppl 1): S21-S23.

22. Gimson, A.E.S., White, Y.S., Eddleston, L.W.F. \& Williams, R. Clinical and prognostic differences in fulminant hepatitis type A, B, and non-A, non-B. Gut 1983, 24: 1194-1198.

Hepatitis B

23. Cockroft, A. \& Walker, P. Surgeons who test positive for the antigen of hepatitis B should be transferred to low-risk duties. Rev Med Virol 1991, 1: 195-200.

\section{Historical lessons}

24. Grey, M.R. Syphilis and AIDS in Belle Glade, Florida 1942 and 1992. Ann Intern Med 1992, 116: 329-334.

\section{Human herpesvirus type 6}

25. Salahuddin, S.Z., Ablashi, D.V., Markham, P.D. et al. Isolation of a new virus, HBLV, in patients with lymphoproliferative disorders. Science 1986, 234: 596-601.

26. Briggs, M., Fox, J. \& Tedder, R.S. Age prevalence of antibody to human herpesvirus 6. Lancet 1988, 1: 1058-1059.

27. Yamanishi, K., Okuno, T., Shiraki, K. et al. Identification of human herpesvirus-6 as a causal agent for exanthem subitum. Lancet 1988, 1: 1065-1067.

28. Pruksananonda, P., Breese Hall, C., Insel, R.A. et al. Primary human herpesvirus 6 infection in young children. $N$ Engl Med 1992, 326: 1445-1450.

\section{Legionnaires' disease}

29. Bhopal, R., Diggle, P. \& Rowlingson, B. Pinpointing clusters of apparently sporadic cases of Legionnaires' disease. $\mathrm{Br} \mathrm{Med}$ $J$ 1992, 304: 1022-1027.

\section{Malaria}

30. Bradley, D.J. \& Warhurst, D.C. Malaria imported into the United Kingdom during 1991. Commun Dis Rep 1993, 3: R25-R28.

31. Watt, G., Shanks, G.D. \& Phintuyothin, P. Prognostic significance of rises in parasitaemia during treatment of falciparum malaria. Trans $R$ Soc Trop Med Hyg 1992, 86: 359-360.

32. Lewis, S.J., Davidson, R.N., Ross, E.J. \& Hall, A.P. Severity of imported falciparum malaria: effect of taking antimalarial prophylaxis. $\mathrm{Br}$ Med J 1992, 305: 741-743.

33. Greenwood, A.M., Armstrong, J.R.M., Byass, P., Snow, R.W. \& Greenwood, B.M. Malaria chemoprophylaxis, birth weight and child survival. Trans R Soc Trop Med Hyg 1992 , 86: 483-485.

34. Steffen, R., Fuchs, E., Schildknecht, J. et al. Mefloquine compared with other malaria chemoprophylactic regimens in tourists visiting East Africa. Lancet 1993, 341: 1299-1303.

35. Bradley, D. on behalf of a meeting convened by the Malaria Reference Laboratory and the Ross Institute. Prophylaxis against malaria for travellers from the United Kingdom. $\mathrm{Br}$ Med J 1993, 306: 1247-1252.

\section{Meningitis}

36. De Moraes, J.C., Perkins, B.A. \& Camargo, M.C.C. Protective efficacy of a serogroup B meningococcal vaccine in Sao Paulo, Brazil. Lancet 1992, 340: 1074-1078.

37. Durand, M.L., Calderwood, S.B., Weber, D.J. et al. Acute bacterial meningitis in adults. $N$ Engl $J$ Med 1993, 328: 21-28. 
Monoclonal antibodies

38. Wenzel, R.P. Anti-endotoxin monoclonal antibodies - a second look. $N$ Engl J Med 1992, 326: 1151-1152.

39. Warren, H., Danner, R.L. \& Munford, R.S. Anti-endotoxin monoclonal antibodies. $N$ Engl J Med 1992, 326: 1153-1156.

40. Jackson, J.E. Anti-endotoxin monoclonal antibodies. $N$ Engl J Med 1992, 327: 889.

41. Rosenfield, S. Anti-endotoxin monoclonal antibodies. $N$ Engl J Med 1992, 327: 889-890.

42. Wenzel, R.P. Anti-endotoxin monoclonal antibodies. $N$ Engl J Med 1992, 327: 891.

43. Welsby, P.D. Should clinicians accept management responsibility? Postgrad Med J 1992, 68: 671-672.

New technology investigations

44. Tompkins, L.S. The use of molecular methods in infectious diseases. N Engl J Med 1992, 327: 1290-1297.

\section{Notification of infectious diseases}

45. Voss, S. How much do doctors know about the notification of infectious diseases. Br Med J 1992, 304: 755.

46. Department of Health. Review of Law on Infectious Diseases Control: Consultation Document. Department of Health, London, 1989.

\section{Occupational diseases}

47. Dalovisio, J.R., Setter, M. \& Mikota-Wells, S. Rhinoceros' rhinorrhea: cause of an outbreak of infection due to airborne Mycobacterium bovis in zookeepers. Clin Inf Dis 1992, 15: 598-600.

\section{Opportunistic infections in AIDS}

48. De Rossell, R.A., De Duran, R. de J., Rosell, O. \& Rodriguez, A.M. Is Leischmaniasis ever cured? Trans $R$ Soc Trop Med Hyg 1992, 86: 251-253.

49. Hunter, G., Baggshaw, A.F., Baboo, K.S., Luke, R. \& Provic, P. Intestinal parasites in Zambian patients with AIDS. Proc R Soc Trop Med Hyg 1992, 86: 543-545.

50. Chave, J.-P., David, S., Wauters, J.-P., Melle, G.V. \& Francioli, P. Transmission of Pneumocystitis carinii from AIDS patients to other immunosuppressed patients: a cluster of Pneumocystitis carinii pneumonia in renal transplant recipients. AIDS 1991, 5: 927-932.

\section{Perils of research}

51. Weiss, E. \& Strauss, B.S. The life and career of Howard Taylor Ricketts. Rev Infect Dis 1991, 13: 1241-1242.

\section{Polio}

52. Wyatt, H.V., Mahadevan, S. \& Srinivasan, S. Unnecessary injections and paralytic polio in India. Trans $R$ Soc Trop Med Hyg 1992, 86: 546-549.

\section{Prevention of infection}

53. Donahue, J.G., Munoz, A., Ness, P.M. et al. The declining risk of post-transfusion hepatitis C infection. $N$ Engl J Med 1992, 327: 369-373.

54. Dodd, R.Y. The risk of transfusion-transmitted infection. $N$ Engl J Med 1992, 327: 419-420.

55. Shapiro, E.D., Gerber, M.A., Holabird, N.B. et al. A controlled trial of antimicrobial prophylaxis for Lyme disease after deer-tick bites. $N$ Engl J Med 1992, 327: 1769-1773.
Prions

56. Pablos-Mendez, A., Netto, E.M. \& Defendini, R. Infectious prions or cytotoxic metabolites? Lancet 1993, 314: 159-161.

57. Hughes, J.T. Prion diseases (editorial). Br Med J 1993, 306: 288.

\section{Rubella vaccination}

58. Miller, E., Waight, P.A., Vurdien, J.E., Jones, G., Tookey, P.A. \& Peckham, C.S. Rubella surveillance to December 1992. Second joint report from the PHLS and National Congenital Rubella Surveillance Programme. Commun Dis Rep 1993, 3: R35-R40.

\section{Sarcoidosis}

59. Fidler, H.M., Rook, G.A., Johnson, N.McI. \& McFadden, J. Mycobacterium tuberculosis DNA in tissue affected by sarcoidosis. Br Med J 1993, 306: 546-549.

60. Scadding, J.G. Mycobacteria and sarcoidosis. Br Med J 1993, 306: $1269-1270$.

\section{Sexual behaviour}

61. Evans, B.G., Catchpole, M.A., Heptonstall, J. et al. Sexually transmitted diseases and HIV-1 infection among homosexual men in England and Wales. Br Med J 1993, 306: 426-428.

62. Archibald, C.P., Schechter, M.T., Le, T.N., Craib, K.J.P., Montaner, J.S.G. \& O'Shaughnessy, M.V. Evidence for a sexually transmitted cofactor for AIDS-related Kaposi's sarcoma in a cohort of homosexual men. Epidemiology 1992, 3: $203-209$.

\section{Tuberculosis}

63. Pearson, M.L., Jereb, J.A., Frieden, T.R. et al. Nosocomial transmission of multidrug-resistant Mycobacterium tuberculosis. Ann Intern Med 1992, 117: 191-196.

64. Fischl, M.A., Daikos, G.L., Uttamchandani, R.B. et al. Clinical presentation and outcome of patients with HIV infection and tuberculosis caused by multiple-drug resistant bacilli. Ann Intern Med 1992, 117: 184-190.

65. Frieden, T.R., Sterling, T., Pablos-Mendez, A. et al. The emergence of drug-resistant tuberculosis in New York City. $N$ Engl J Med 1993, 328: 521-526.

66. Gorble, M., Iseman, M.D., Madsen, L.A., Waite, D., Ackerson, L. \& Horsburgh, R. Treatment of 171 patients with pulmonary tuberculosis resistant to isoniazid and rifampin. $N$ Engl J Med 1993, 328: 527-532.

67. Dooley, S.W., Jarvis, W.R., Martone, W.J. \& Snider, D.E. Multidrug-resistant tuberculosis. Ann Intern Med 1992, 117: 257-258.

68. Wilson, S.M., Nava, E., Morales, A., Godfrey-Faussett, P., Gillespie, S. \& Andersson, N. Simplification of the polymerase chain reaction for detection of Mycobacterium tuberculosis in the tropics. Trans R Soc Trop Med Hyg 1993, 87: $177-180$.

\section{Vaccination}

69. Cooke, B. Immunisation, not vaccination. Br Med J 1992 304: 1313.

70. Anonymous. Typhoid vaccination: weighing the options. Lancet 1992, 340: 341-342.

Vitamin A

71. Glasziou, P.P. \& Mackerras, D.E.M. Vitamin A supplementation in infectious diseases: a meta-analysis. Br Med J 1993, 306: $366-370$

\section{Whooping cough}

72. Khattak, M.N., Matthews, R.C. \& Burnie, J.P. Is Bordetella pertussis clonal? Br Med J 1992, 304: 813-815. 